\title{
COMPRENSIÓN LECTORA Y UNA VISIÓN DE CAMBIO EN LA FORMACIÓN DE ADMINISTRADORES DE EMPRESAS
}

\author{
READING COMPREHENSION AND A VISION OF CHANGE IN FORMATION OF \\ DIRECTORS OF COMPANIES
}

\section{LEITURA E UMA VISÃO DE MUDANÇA NA FORMAÇÃO DE GESTORES DE EMPRESAS}

\author{
Por: ZEVALLOS PEÑA_Federico Manuel, CORREDOR GARCÍA_ María del Pilar
}

\begin{abstract}
Magister en Ingeniería Industrial, Universidad de los Andes, Bogotá. Profesor Facultad de Ciencias Económicas y Administrativas, Universidad Jorge Tadeo Lozano. Email: manuel.zevallos@utadeo. edu.co, federicozp@hotmail.com, Colombia.

Magister en Administración de Empresas de la Universidad Pablo de Olavide, Sevilla, España. Profesora Facultad de Ciencias Económicas y Administrativas, Universidad Jorge Tadeo Lozano. Email: mariap.corredor@utadeo.edu.co, Colombia.
\end{abstract}

DOI: http://dx.doi.org/10.22267/rtend.171802.82

\section{RESUMEN}

El objetivo de este artículo de reflexión es establecer el aporte de las competencias en comprensión lectora, en el logro de una visión más amplia y dinámica de los administradores de empresas en la interpretación de los contextos en los cuales se desenvuelven las organizaciones. El artículo incluye: 1) una presentación del enfoque de las cuatro dimensiones filosóficas de Bedard, (Praxeología, Epistemología, Axiología y Ontología), 2) la Lectura, definiciones y sus funciones, 3) la lectura como "valor", 4) una descripción de los principales aspectos de la formación tradicional en administración según Omar Aktouf, 5) la propuesta, del mismo Aktouf de un enfoque formativo basado en lo que el autor denomina una "comprensión crítica permanente". Se finaliza con un análisis sobre los aportes de la comprensión lectora en la formación de en administración, desde las capacidades desarrolladas en las dimensiones filosóficas. Se concluye que las capacidades en comprensión lectora contribuyen a la formación de una "Comprensión Crítica Permanente" por parte de los administradores, y con ello, a una mejor interpretación de los contextos, dinámicas y agentes en los que se desenvuelven las organizaciones, lo cual a su vez contribuirá a una mejor toma de decisiones por parte de los administradores. 
Palabras clave: comprensión de lectura, dimensiones filosóficas, valores de la lectura, formación en administración, comprensión crítica permanente.

JEL: I21, A22, L21.

\section{ABSTRACT}

The aim of this article is to make a reflection of contribution of skills in reading comprehension, in achieving a broader vision and dynamics on business managers interpretation of contexts in which organizations operate. This is an argumentative article, which is based on the theore ${ }^{1}$ tical framework provided, that includes: 1) a presentation of the approach of "Four Philosophical Dimensions" of Bedard 2) Reading definitions and functions, 3) Reading as "a value", 4) a description of Omar Aktouf vision about traditional management education and its main aspects, 5) a description of an Aktouf education management approach proposal, based on "permanent crítical understanding". This article ends analyzing contributions of reading comprehension in business administration education and enables a wider dynamic vision of contexts in which organizations operate. It concludes that capabilities in reading comprehension are important for the formation of what Aktouf called an "permanent crítical understanding" by managers, and thus to a better understanding of the contexts, dynamics and agents in which organizations operate, which in turn will contribute to better decision-making by managers.

Keywords: reading comprehension, philosophipal dimensions, value of reading, management education, permanent crítical understanding.

JEL: I21, A22, L21.

\section{RESUMO}

O objetivo deste artigo é o de estabelecer a reflexão contribuição de habilidades em compreensão de leitura, para alcançar um gerentes de negócios mais amplos e dinâmicos na interpretação dos contextos em que as organizações operam visão. O artigo inclui: 1) a abordagem de apresentação dos quatro dimensões filosofia de Bedard, (Praxeologia, Epistemologia, Axiología ontologia), 2) de leitura, as definições e funções, 3) leitura como "valor", 4) um descrição dos principais aspectos da gestão da formação tradicional, Omar Aktouf, 5) a proposta, a mesma Aktouf de uma abordagem de gestão de treinamento com base no que o autor chama de "compreensão crítica permanente." Ele termina com uma análise das contribuições da compreensão da leitura na formação de administração, uma vez que as capacidades desenvolvidas em dimensões filosóficas. Conclui-se que a capacidade de compreensão de leitura contribuir para a formação de uma "compreensão crítica Standing" por parte dos gestores, e, assim, para uma melhor compreensão de contextos, dinâmicas e agentes em que as organizações operam, o que por sua vez, contribuirá para uma melhor tomada de decisão pelos gestores.

1 Análisis de la Educación; Enseñanza universitaria; Objetivos de negocio de la Empresa. 
Palavras-chave: compreensão de leitura, dimensões filosóficas, valores de leitura, formação em gestão, crítica entendimento permanente.

JEL: I21, A22, L21.

\section{INTRODUCCIÓN}

Los fallos y casos de corrupción en la gestión de grandes corporaciones que han causado las crisis financieras recientes a nivel global, se consideran razones suficientes para motivar una reflexión sobre la formación impartida en las escuelas de administración, y en particular, para indagar sobre el nivel y uso de competencias genéricas ${ }^{2}$, como el pensamiento crítico, que hacen los administradores en la toma de decisiones de las organizaciones.

En estos contextos, donde son frecuentes los cuestionamientos a los enfoques tradicionales en la formación en administración y su capacidad para desarrollar competencias genéricas, cuando cobran especial importancia dichas competencias como fuentes de formación de pensamiento crítico en los administradores. La capacidad de comprensión lectora ${ }^{3}$ aparece como una de las competencias genéricas indispensables para el ejercicio profesional, y su importancia resulta fundamental en el tránsito y desarrollo social de los individuos, además del reconocimiento del contexto que lo rodea. El presente artículo hace énfasis en la capacidad de comprensión lectora, y el ejercicio de la lectura como fuentes para el desarrollo de una "comprensión crítica permanente" que permita a los administradores una mejor toma de decisiones.

A continuación se presentan los temas a considerar en el artículo:

- Se presenta el enfoque de las dimensiones filosóficas de Bedard, sobre la formación y praxis en administración, y las "dimensiones filosóficas" involucradas en estos procesos (Praxeología, Epistemología, Axiología y Ontología).

- Se describen los procesos asociados al ejercicio de la lectura y se señala el valor de la lectura:

○ Como "medio" para la adquisición de información y conocimientos disciplinares.

○ Como "fin" en sí mismo, para el análisis de contenidos desde los supuestos, argumentos y conclusiones.

\footnotetext{
2 Las "competencias genéricas" se definen como “... aquellas que resultan indispensables para el desempeño social, laboral y cívico de todo ciudadano” (Bernal y Mariño, 2013). Es por esta razón que las competencias genéricas también se denominan como "competencias para la vida", ya que "no pierden relevancia, ni siquiera en educación superior", debido a que su ejercicio y desarrollo contribuyen a facilitar ese "desempeño social".

3 La comprensión lectora se entiende como "la capacidad de leer comprensivamente diversos tipos de textos, mediante la aplicación de estrategias comunicativas y lingüísticas específicas que posibilitan el análisis y el establecimiento de relaciones entre los distintos componentes que conforman un texto".
} 
- Se exponen dos enfoques presentados por Omar Aktouf para la formación en administración: el enfoque tradicional actual, de reproducción y doctrinario; y el enfoque de comprensión crítica permanente que permite una visión más amplia y dinámica del contexto de las organizaciones.

- Se articulan las contribuciones de la lectura y su valor (como 'medio' y 'fin') en los procesos formativos, con el desarrollo de una capacidad de comprensión crítica permanente (Aktouf, 2000) en los administradores.

- Se recogen los aportes principales en las conclusiones del artículo.

\section{FUNDAMENTOS TEÓRICOS (REVISIÓN DE LITERATURA)}

\section{II.1 Bedard y las cuatro dimensiones filosóficas.}

Renee Bedard (del grupo HEC de Montreal), propone un modelo para el estudio de un conjunto de "cuestiones" fundamentales en administración como son:

- "El lugar del ser humano en el campo de los estudios y de las prácticas administrativas", y

- $\quad$ "Los fundamentos teóricos del pensamiento y el conocimiento administrativo"4.

Dicho modelo consiste en reconocer cuatro dimensiones filosóficas las cuales interactúan y se encuentran comprometidas en cualquier proceso de "comprensión seria de la realidad" (procesos formativos, interpretación de contextos, etc.). Estas son las dimensiones Praxeológica, Epistemológica, Axiológica y Ontológica.

Dimensión Praxeológica: Bedard inicia la presentación de las dimensiones del modelo con la dimensión praxeológica, la cual asocia a la definición de "praxis" planteada por Aguirre (2002) como "la actividad humana material y social de transformación de la realidad objetiva de la naturaleza, de la sociedad y del hombre mismo"; para luego aclarar (Bedard) que utilizará una definición de praxeología desde "un punto de vista general ... en un sentido más amplio", el cual abarcará "todos los aspectos de la actividad humana y no solamente la acción y sus diferentes realidades particulares". ${ }^{5}$

Bedard menciona también que la observación de situaciones y fenómenos concretos suministran información ligada a la sensibilidad proveniente de los cinco sentidos y los sentimientos. Esta información debe ser "evaluada" con la finalidad que ella avance

\footnotetext{
4 Bedard incluye también el estudio de "las especificidades de Occidente y su administración" como una tercera "cuestión" en la presentación de su modelo de dimensiones filosóficas.

5 Bedard señala como aspectos comprendidos dentro de la Praxeología: "las diferentes conductas humanas y las actividades de creación, de producción y de fabricación (la praxis y la poiesis); las actitudes (en los planos cognitivos, afectivos y comportamentales); el saber hacer y las habilidades; las prácticas, los métodos de trabajo y los procedimientos; las herramientas, los materiales, las técnicas y la tecnología; las palabras y los escritos; los productos y los resultados".
} 
en su grado de certidumbre, la cual comprende dos tipos de certezas diferentes pero complementarias, que se encuentran vinculadas a otras dos dimensiones filosóficas propuestas en el modelo de Bedard:

- La Validación, de naturaleza intelectual, que trata sobre la exactitud, la confirmación o la verificación y, como tal, de dominio de la Epistemología, donde el "elemento clave es el criterio de validez". Es así como una cosa es considerada "válida" cuando "ha sido revestida de todas las formalidades necesarias en el momento presente", de tal forma que "no hay nada que agregar para que tenga toda su fuerza". Por ello, la validez "implica la idea de valor absoluto, esencial, reconocido sin condición en todas partes", la cual está asociado a una "normatividad científica, impersonal y objetiva" que lleva a "juicios científicos".

- $\quad$ La Legitimación, de orden moral, que se interesa en la justificación según el buen derecho, la razón, el buen sentido, la cultura, la moralidad, de dominio de la Axiología, donde el elemento clave es el criterio de valor. Es así como una cosa es considerada como "valedera" cuando tiene un valor "relativo, admisible solamente en ciertos casos, con relación a ciertas personas o ciertas cosas", el cual se encuentra asociado a la voluntad y subjetividades del sujeto que lleva a los llamados "juicios axiológicos o del juicio de valor".

Dimensión Epistemológica: Sobre la epistemología, Bedard menciona que esta se interesa "tanto en la teoría de la ciencia en general... como en el estudio de las ciencias consideradas como las realidades que se observan, describen y analizan". Bedard habla también de una "competencia epistemológica" citando a Chanlat (1984) cuando afirma que para la administración "bajo su doble aspecto de ciencia y arte, de conducta y de acciones creadoras, de administración de las cosas y de gobierno de las personas", estos aspectos representan a su vez "la puesta en práctica de los conocimientos adquiridos empíricamente, los procedimientos inéditos y las concepciones teóricas reconocidas”. Aunque la definición anterior sugiere que la epistemología representa únicamente el conocimiento asociado a "una disciplina o un campo especializado", esta involucra también una "función general de vigilancia crítica que ejerce su autoridad" sobre dicha disciplina.

Dimensión Axiológica: Bedard especifica la axiología como el campo de los valores individuales (morales) y colectivos (culturales), así como los principios que determinan las costumbres. Bedard es específica cuando afirma que esta dimensión "comprende la ética ${ }^{6}$ y la moral". Bedard señala también que "la axiología ejerce una función de vigilancia crítica respecto a la praxeología para asegurar su legitimidad".

Dimensión Ontológica: Bedard inicia la presentación de esta dimensión citando a Dardel (1954), cuando menciona que, en el individuo, "la ontología determina su

6 Sobre la "Ética", Bedard señala que ésta "se interesa en los principios generales de la conducta humana y tiene por objeto la teoría de la acción moral, individual y colectiva". A partir de la ética se crean la política (para la vida en sociedad), la deontología (para la vida en el trabajo) y la moral doméstica (para la vida en familia).

7 Sobre la "Moral", Bedard afirma que esta "se entiende como un conjunto de reglas que se imponen a la conducta de cada hombre en la sociedad donde vive y en su vida privada". 
'manera' de habitar lo real, de orientarse en medio de las cosas y de encontrar una salida en la búsqueda de si (mismo)". En este mismo sentido, Bedard afirma que "la ontología suministra las grandes ideas directrices que permiten a cada uno orientarse, asegura la inteligibilidad de base y procura las certidumbres fundamentales... (Para ofrecer)... una cierta lectura del universo, una primera concepción de la realidad". Bedard resume estas ideas cuando afirma que "ontología es la matriz y la base sobre sobre la cual todo edificio conceptual (epistemología), ético (axiología), crítico y práctico (praxeología) de cada persona es subsecuentemente construido".

Bedard representa gráficamente estas dimensiones en los vértices de un rombo. En ella, la dimensión Praxeológica aparece en el vértice superior dado que Bedard la asocia con el actuar visible del individuo en la organización y el contexto. Bedard también establece una analogía de dicha figura con un iceberg, al señalar la punta del iceberg en el vértice superior del rombo como el elemento "visible" y resultante de las otras tres dimensiones, y de sus interacciones.

Figura 1. Las cuatro dimensiones filosóficas - el "Rombo" de Bedard PRAXEOLÓGICA

EPISTEMOLÓGICA

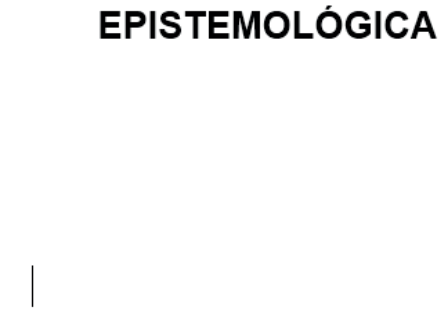

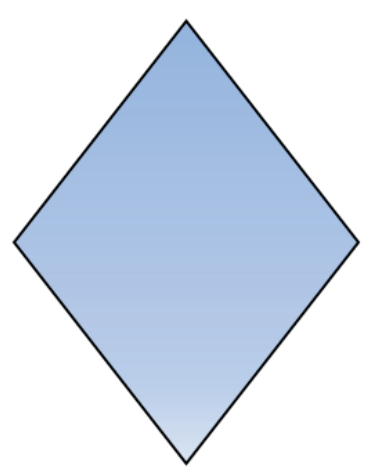

\section{ONTOLÓGICA}

Fuente: Bedard (2003).

\section{AXIOLÓGICA}

\section{II.2 La lectura - algunas definiciones y procesos}

El valor de la lectura es algo que pocos pueden discutir. Aparecen muchos beneficios resultantes del ejercicio de la lectura que han sido citados en diversos artículos, tanto de académicos como de opinión. Es por ello que a continuación, se profundizará sobre la lectura, sus definiciones y los procesos asociados a ella.

Se considera la lectura como un proceso constructivo de comunicación e interactivo, el cual incluye tanto procesos de decodificación (microprocesos), como de comprensión (macroprocesos) (Paredes, 2006). 
Se dice que es "constructiva" porque el lector, al procesar el texto como lenguaje e interiorizarlo, construye su propio significado (Morales, 2002). El mismo autor hace una diferencia entre "información" y "conocimiento", al señalar que la transformación de la "información" y su apropiación en forma de "conocimiento" demanda tiempo y reflexión; este último proceso es el que finalmente permite al lector comprender y asimilar dicha información.

A su vez, se dice que la Lectura es "interactiva" porque ella constituye un proceso complejo de interacción entre el lector y el texto (Solé, 1999). Según este mismo autor, es durante esta interacción que el lector, que busca satisfacer un conjunto de objetivos (aquellos que guían y justifican su lectura), realiza una intervención "antes, durante y después" del acto lector, las cuales se encuentran vinculadas con la relación entre "leer, comprender y aprender".

Sobre el "Significado" de un texto, Morales (Morales, 2002) afirma que éste no constituye una propiedad del texto sino de la interpretación que le otorga el lector, quien lo construye en un proceso de transacción flexible el cual consiste en que, conforme se va leyendo, el lector le va otorgando un sentido particular al texto.

A su vez Lerner (Lerner, 1985) señala que la interpretación y la construcción del significado del texto se da a partir de un conjunto de capacidades, experiencias e intereses del lector como son sus competencias comunicativas, conocimientos previos, concepción del mundo, experiencias previas en lectura y escritura y el propósito de la lectura.

Cabe destacar también lo afirmado por Kintsch (1989) y Perfetti (1989) (citados por Echevarría y Gastón, 2000), cuando señalan que la comprensión de un texto exige la puesta en práctica de una capacidad (compleja) que permita la construcción de un modelo del mundo o de la situación descrita en el texto.

Es así como la lectura, desde un contexto proporcionado por los conocimientos previos y los objetivos de lectura de una persona, puede dar lugar a que la representación que se haga del contenido de un texto y, con ello, el modelo de situación que construya, varíe de una persona a otra. Es así como un mismo texto puede dar lugar a la realización de inferencias diferentes (Echevarría y Gastón, 2000). 
Figura 2. Lectura: proceso de construcción de significado

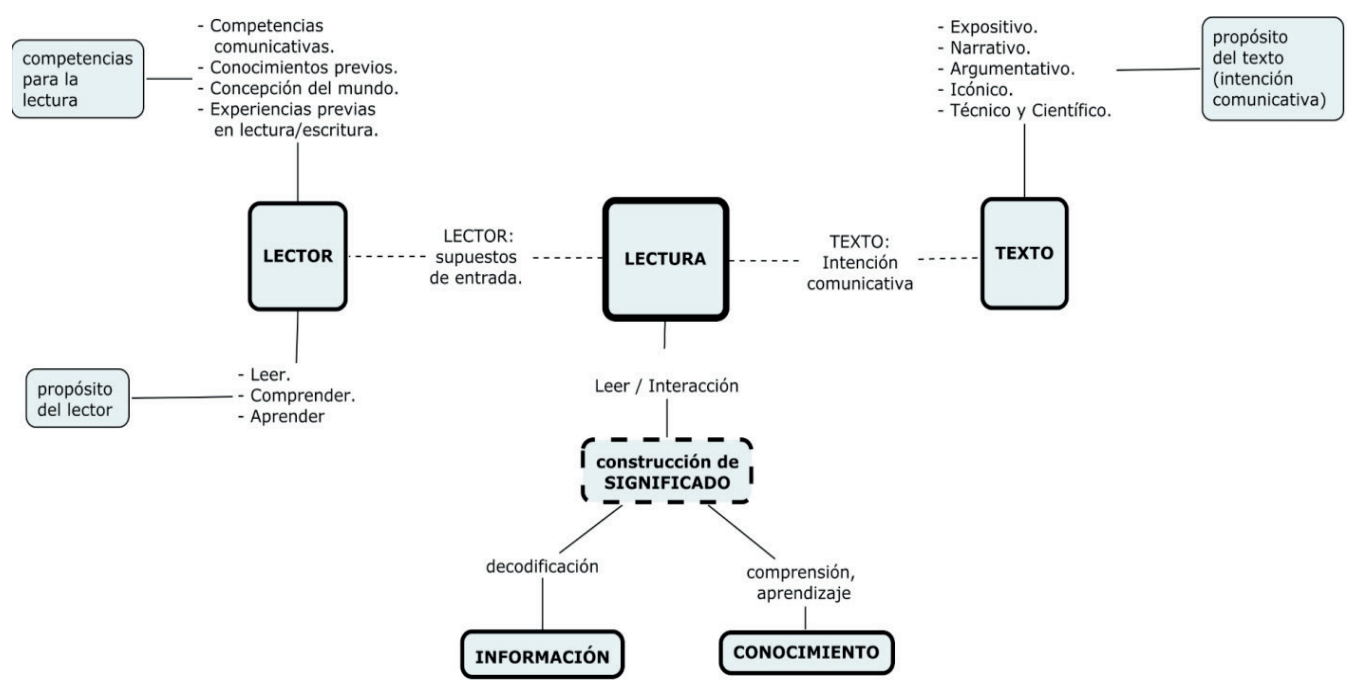

Fuente: Elaboración propia.

En la Figura 2 se propone un ordenamiento de los elementos expuestos sobre la Lectura, los actores y sus características y el proceso de construcción de significado. Se aprecia que aparecen dos elementos básicos en el proceso de lectura, que son el lector y el texto, los cuales presentan un conjunto de supuestos "de entrada", o previos al acto de la lectura. Estos supuestos de entrada del lector lo constituyen las competencias para la lectura por parte del lector, así como sus propósitos de lectura. De manera similar, el texto a ser leído también tiene un propósito o intención comunicativa, definida por el autor del texto. Es así como, a partir del proceso de lectura y de los supuestos de entrada mencionados anteriormente, se logra la construcción del "Significado" por parte del lector, quien lo apropia en la forma de información, o de conocimientos.

\section{II.3 La lectura como "valor"}

Reboul aproxima una definición de "Valor", cuando afirma que "todo valor se define por el sacrificio" (Reboul, 1992). El "valor" de algo que queremos obtener constituye un atributo de ese "algo" físico (objeto) o abstracto (aspecto), que "aparece en el momento en que ese 'algo' deja de sernos indiferentes, y nos atrae"; es por ello que consideramos que vale la pena hacer algún sacrificio para obtenerlo. Es así como el "valor" de ese "algo" se interpreta como equivalente a aquello que estamos dispuestos a sacrificar para obtenerlo.

Desde estas definiciones e interpretación previa, el mismo Reboul plantea dos funciones para la lectura según el valor de lo que es posible obtener de ella: un valor de la lectura como "medio" y como "fin" en sí misma. 
Como "medio", cuando la lectura puede ser utilizada como un "medio" de apropiación (comunicación) de datos (información) o de conocimientos (aprendizaje). Para cualquiera de estos casos, se trata de la función utilitaria de la lectura, la cual supedita su valor al beneficio que podamos obtener con la información o los conocimientos obtenidos del texto consultado.

Sin embargo, Reboul destaca también el valor propio ('per se') de la lectura "como fin" en si misma, cuando afirma que en el acto de leer se da "esa unión indisoluble de lo sagrado (religioso) y la razón" (Reboul, 1992). Sobre esta última afirmación, dicho autor hace una descripción de lo que representa cada uno de estos componentes que se encuentran en el acto de leer:

- Un acto "Religioso", porque se aborda el texto "con el mismo respeto, la misma expectación, la misma esperanza que el creyente". Como lectores nos volvemos disponibles y abiertos al mensaje desde la suposición que el texto tiene un sentido que se descubre según la lectura y comprensión del lector. Es así como "se aprende lo que no se sabía”, y uno "se convierte en lo que no era". Reboul sintetiza este "carácter religioso" al afirmar que lo "sagrado" representa "no admitir nunca que un texto no tenga sentido" (Reboul, 1992).

- Y Y un acto "Crítico", porque "aquel que publica un texto, lo somete al juicio de lectores, "quienes comprueban la solidez de los argumentos, los supuestos de sus enunciados y lo que (en él) queda implícito" (Reboul, 1992). En este caso, se ejercita la razón al "desmenuzar (el texto) hasta haber encontrado ese sentido para luego, una vez comprendido, releerlo bien varias veces". El simple hecho de leer, de tener el mensaje ante los ojos como un objeto, libera al lector de tener que identificarse con el mensaje, pues el pensamiento lo presenta al texto y su significado, como un objeto que es posible tomar, dejar y retomar a nuestro antojo, sin estar obligados a creer en él. La lectura le permite al lector "juzgar, críticar, reflexionar y comprender, sin tener que aceptarlo todo de manera incondicional".

Figura 3. La lectura como "medio" y como "fin"

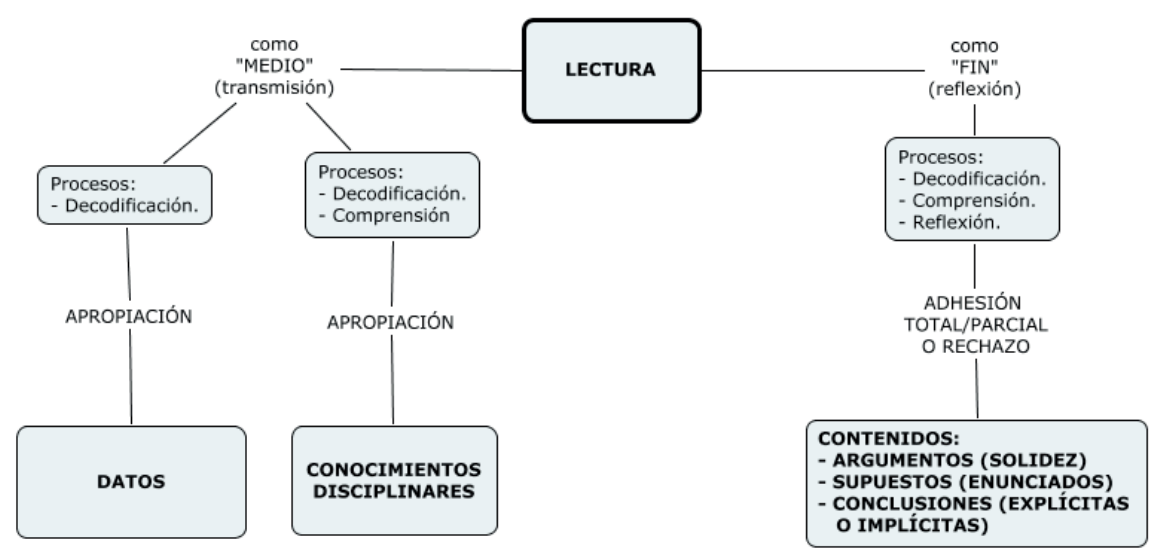

Fuente: Elaboración propia. 
En la Figura 3 se sintetiza lo expuesto en el punto anterior, donde se presentan dos de las funciones (valores) principales de la lectura: (1) como "medio" de transmisión de datos o de conocimientos, los cuales incluyen a los conocimientos disciplinares que forman parte de un proceso formativo (por ejemplo, en la educación superior), y (2), como "fin" en sí misma, que en la interacción entre el lector y el texto genera, además de los procesos necesarios requeridos en la función anterior (decodificación, comprensión), la "reflexión" que hace que el lector se adhiera total o parcialmente, o incluso rechace, los argumentos, supuestos y conclusiones contenidos en el texto leído.

Es así como la lectura en su funcionalidad de "medio" representa un instrumento que contribuye a incrementar capacidades de tipo "epistemológico" del lector, en la medida que se aborden textos cuyos propósitos sean tanto de tipo disciplinar como multidisciplinar. Con ello, el lector puede lograr niveles de competencia disciplinares y multidisciplinares que le permitan además ejercer una "vigilancia crítica" de las actividades (praxeología) y garantizar su "validez" según la disciplina correspondiente.

De otra forma, al enfrentar el lector a textos de contenido expositivo, narrativo y argumentativo la lectura, en su funcionalidad como "fin" en sí misma, hará que el lector realice procesos de análisis y reflexión que lo lleven a emitir "juicios axiológicos o de valor" coherentes para legitimar (o no) las ideas o situaciones expuestas en los textos. Ello motivará dinámicas y ejercicios de tipo "axiológico" que ayudarán a la construcción (o deconstrucción) de una ética o una crítica formada desde dichas reflexiones.

\section{II.4 Aktouf y la enseñanza en Administración}

En este punto nos introducimos al análisis desarrollado por Omar Aktouf sobre los enfoques predominantes de enseñanza - aprendizaje en los programas de administración de empresas y escuelas de negocios. Este autor considera dos tipos de formación:

(I) Una formación tradicional, orientada hacia la reproducción del conocimiento disciplinar impartido y, con ello, la consolidación de las condiciones del momento histórico actual y sus características dominantes, y

(II) Un segundo tipo de formación que, reconociendo la tradición en administración (estado de la disciplina), permite y fomenta una aproximación y apropiación crítica de dicha tradición: una "comprensión crítica permanente".

Figura 4. La Administración: ¿orientada a la "reproducción”, o al "cambio"?

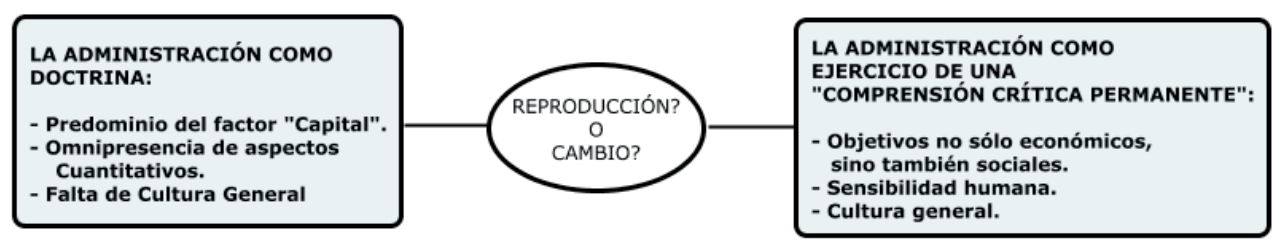

Fuente: elaboración propia. 


\section{II.4.1 El enfoque tradicional o de reproducción.}

El primer enfoque, "la administración como doctrina”, el cual Aktouf considera que es el que prevalece en la mayoría de escuelas de negocios, se fundamenta en:

- La reproducción de modelos y modos de pensamiento o "doctrinas" de un grupo de empresarios que las comparten, las cuales constituyen el fundamento de la mayoría de los contenidos curriculares de los programas;

- Se considera además que estos empresarios representan la clase conservadora de las economías occidentales, dado que son estos los que se benefician con la continuidad de un momento histórico favorable a los intereses y al creciente lucro de las corporaciones que estos representan.

Aktouf (2000) menciona tres ideas que soportan esta orientación conservadora y tradicionalista en la formación de los administradores.

(1) "La definición estrecha de la administración, centrada en el predominio del factor capital y en cuidado del enriquecimiento individual".

El mismo autor considera estos contenidos en su mayor parte como tradicionales, dirigidos con un énfasis doctrinal y con una visión crematística de la economía y las organizaciones, en la que se privilegia al factor capital ubicándolo como el factor más importante, valioso y por lo tanto, como el único cuya minimización de riesgo constituye la labor más importante en cualquier organización, muy por encima de otros factores de los procesos productivos o de servicios, como lo son el trabajo y los recursos naturales.

(2) "La omnipresencia de aspectos cuantitativos, en particular del cálculo económico".

Aktouf también hace énfasis en que este enfoque tradicional incentiva el predominio de cálculos, resultados numéricos y el uso de instrumentos matemáticos, lo que ha llevado a convertirlas en herramientas preponderantes (casi exclusivas) en la toma de decisiones de los administradores de empresa actuales. Es así como,

- la formalización de estos énfasis en los currículos de formación en administración conlleva nuevamente a “... abstracciones (modelos) de la realidad que favorecen unas condiciones a priori establecidas en estos modelos numéricos; además de

- $\quad$ una recurrente dependencia de la computadora y de una automatización, en detrimento "de la inteligencia, de la sensibilidad, de la intuición" para la toma de decisiones (Aktouf, 2000).

(3) "La falta de cultura general en la formación del administrador." 
Aktouf afirma que el enfoque tradicional de formación en administración establece como uno de sus principales paradigmas que el administrador debe ser un personaje de acción, de certezas e incluso automatismos que conlleven a una rápida toma de decisiones asistidas en su mayor parte por instrumentos y cálculos. En este mismo sentido, el autor considera que la mayoría de escuelas de negocios son reacias a la inclusión de temas de formación en cultura general y en humanidades, dado que el enfoque tradicional busca privilegiar contenidos para una actuación y toma rápida de decisiones, y no para la reflexión con temas y lecturas que motiven preguntas y discusiones cuestionadoras.

\section{permanente}

\section{II.4.2 El enfoque de cambio o de ejercicio de la comprensión crítica}

Es así como encontramos que el enfoque tradicional presenta una fortaleza epistemológica en los conocimientos disciplinares que, como se mencionó anteriormente, son de tipo doctrinal y destinados a ser reproducidos para consolidar las condiciones principales del momento histórico actual, que son las que favorecen los intereses de la élite que sustenta dicha doctrina.

Algunos de los inconvenientes de este enfoque de formación en administración es que resulta cada vez más frecuente encontrar casos de empresas que, en la praxis de sus valores misionales, hacen primar aquellos valores asociados a la misión económica de la empresa (ser rentables, dar beneficios) muy por encima de aquellos otros relacionados a la misión social general de la empresa y, por ende, que se encuentran en clara relación con los valores éticos o compartidos con la sociedad (coherencia, equidad en las cargas y beneficios, participación y consenso, respeto y mejora del medio ambiente, etc.). Lo anterior se manifiesta en casos de empresas que atentan contra el bienestar social, la igualdad y la ecología, al ejecutar acciones que van desde la consolidación de economías de consumo y especulativas, incluyendo la liberalización financiera sin restricciones que permite la libre entrada y salida de los capitales internacionales, pasando por la depredación del medio ambiente, hasta el uso de la precariedad y la temporalidad laboral como fuentes de rentabilidad (Diez Gutiérrez, 2009).

Frente a lo anterior, Aktouf reclama una formación en administración que fomente el ejercicio de una "comprensión crítica permanente" en los administradores. Sobre dicho enfoque, el autor señala algunas de sus principales características:

- La formación en administración debe ir mucho más allá de la simple reproducción de doctrinas, dada la necesidad que ésta (la administración) se integre a un proyecto social mucho más amplio.

- La administración, entendida como una disciplina "híbrida", debe agrupar además de los conocimientos teóricos, unas prácticas que proporcionen objetivos no solo económicos sino también sociales.

- Ello conlleva a un llamado a: 
- Despertar la sensibilidad personal de los estudiantes,

- A ofrecerles una mayor cultura que posibilite en los estudiantes una actitud de análisis y cuestionamientos a lo largo de la enseñanza de la disciplina.

Para lo anterior, se requeriría una serie de cualidades resultantes de la formación en administración que trasciendan el enfoque tradicional de formación en administración.

\section{APORTES DE LA LECTURA EN LA FORMACIÓN EN ADMINISTRACIÓN}

Respecto al enfoque tradicional en la formación en administración, para la primera característica,

(1) Aktouf reclama de los estudiantes, profesionales y de los profesores en administración de empresas, una actitud crítica frente a los contenidos curriculares propuestos por las escuelas de administración.

Es aquí donde vinculamos a esta perspectiva la importancia de la lectura, entendiendo que dicha crítica sólo adquiere sentid o y trascendencia cuando proviene del reconocimiento de la importancia de los demás factores de producción (y los stakeholders involucrados en dichos factores) y del cuestionamiento de la importancia del factor Capital en el enfoque tradicional existente.

Se propone la lectura como una fuente de pensamiento y actitud crítica desde dos enfoques:

- Uno cuantitativo, a partir de la lectura de una cantidad mayor de textos disciplinares, con el consiguiente fortalecimiento en lo epistémico.

- Otro enfoque cualitativo, desde una lectura reflexiva de los conocimientos disciplinares que la someta a cuestionamientos reflexionados. En otras palabras, una lectura acompañada de un proceso reflexivo que ejercite y desarrolle las dimensiones axiológica (ética y valores) y ontológica (razón de ser del individuo y su lugar en la organización y comunidad).

Lo anterior, complementado con la inclusión de textos disciplinares e información (casos), que motiven un ejercicio previo de interpretación, que lleven a discusiones, debates y la adopción de posturas provenientes de consensos (o disensos) desde un ejercicio argumentativo y discursivo.

Para la característica (2), que destaca la omnipresencia de los aspectos cuantitativos en la formación y praxis de los administradores de empresas para una rápida toma de decisiones, se propone también la lectura como un ejercicio de reflexión que al cultivarse a lo largo del proceso formativo sirva como complemento al uso de instrumentos y de los juicios matemáticos resultantes (muchas veces carentes de valor 'per se') para una interpretación y comprensión más integral de la realidad, sus diversos actores y los impactos resultantes. 
Lo anterior ayudaría a entender el uso de los instrumentos y modelos cuantitativos como un elemento más a considerar dentro de los insumos disponibles para una mejor toma de decisiones, dentro de los cuales debemos incluir las reflexiones mencionadas anteriormente. Esto último daría también fortalezas en el aspecto epistemológico del administrador.

Finalmente para la (3) característica, que resalta la falta de cultura general en el enfoque tradicional en la formación en administración, la importancia de la lectura para subsanar esta falencia es explícita, cuando se reclama que el administrador debe ser un lector constante e inagotable no sólo de los contenidos correspondientes a su disciplina, sino de las humanidades y también de las artes, con la finalidad que el administrador sea capaz de "integrar, gracias a su cultura general, experiencia o intuición, diferentes disciplinas y conocimientos, tanto en el terreno de la gestión general como en el de las ciencias humanas".

Aquí aparece el valor de la lectura como "instrumento", para integrar un mayor conocimiento no solo de la epistemología de las disciplinas incluidas en la administración (administración, mercadeo, economía, producción, etc.), sino también proveniente de las ciencias sociales (sociología, psicología, educación, etc.), lo cual le permitirá al administrador realizar análisis e interpretaciones y comprensiones desde diversas perspectivas integradas o interdisciplinarias.

- A su vez, se espera que al promover de esta forma la adquisición de "conocimientos más finos y diversificados", estos se traduzcan en "una mayor sensibilidad en los aspectos humanos, ecológicos y culturales de la empresa” por parte de los administradores (Aktouf, 2000).

- $\quad$ En este punto, el aporte de la lectura de contenidos asociados con las humanidades y las artes se da como medio para el acercamiento a los diversos géneros literarios (narrativo, dramático, lírico, etc.). Para estos contenidos se espera que el valor de la lectura deje de ser estrictamente instrumental, para trascender a reflexiones que lleven al desarrollo de esta "sensibilidad" los cuales influencian no sólo la epistemología del individuo, sino también su axiología y ontología.

Según lo anteriormente expuesto, se espera que lo anterior posibilite una nueva visión de las situaciones y contextos a los que se enfrentan los administradores y las organizaciones, y que el desarrollo de competencias lectoras en la formación de administradores lleve a una mejor comprensión de los fenómenos y dinámicas sociales; además del reconocimiento del individuo y su complejidad, como elemento necesario y central de cualquier organización.

La exposición hecha por Aktouf sobre el desbalance que existe entre los dos tipos de formación, reproducción y cambio, es congruente con el momento histórico actual donde aparece en clara ventaja el primero. Pero esta situación puede ser transformada si se acude a la una comprensión crítica permanente, como parte de las competencias genéricas que deben ser atendidas y fomentadas por las Escuelas de Administración. Y la lectura, su práctica y el desarrollo de capacidad de comprensión lectora resultan fundamentales no 
sólo desde su función utilitaria, sino desde su capacidad para generar procesos reflexivos profundos en el lector que permitan esta comprensión crítica.

\section{CONCLUSIONES}

- $\quad$ El valor de la lectura se encuentra asociado a sus funcionalidades:

- Como un "medio" de apropiación de datos y conocimientos disciplinares (epistemología),

- Así como un "fin" en sí misma, a partir del análisis y la reflexión derivadas de su práctica y del contenido del texto.

- El enfoque tradicional de formación en administración se orienta hacia la reproducción del conocimiento disciplinar, lo cual lleva a consolidar las características dominantes del momento histórico actual. Este enfoque se fundamenta en fortalezas de tipo "epistemológicas" en conocimientos disciplinares de tipo doctrinal.

- $\quad$ Aktouf propone un enfoque de "comprensión crítica permanente" en la formación en administración que trascienda la mera reproducción de la disciplina. Con ello se espera que los estudiantes se encuentren en condiciones de apropiar conocimientos de tipo multidisciplinarios así como de cultura general, que promuevan una actitud de análisis y cuestionamiento a lo largo del proceso formativo.

- Las fortalezas derivadas de la práctica de la lectura y el consiguiente desarrollo de fortalezas en comprensión lectora pueden contribuir al desarrollo de una "comprensión crítica permanente" en la formación en administración:

- Como facilitadores en los procesos formativos de los estudiantes para la apropiación de conocimiento. Ello se lograría desde la función de la lectura como "medio" para la apropiación tanto de conocimientos formativos, disciplinares, así como multidisciplinares. Con lo anterior, el administrador desarrollaría fortalezas de tipo epistemológicas.

- Como inductores de una actitud crítica que promuevan procesos de reflexión. Ello se lograría desde la función de la lectura como "fin" en sí misma, que posibilite procesos de reflexión que cuestionen los conocimientos disciplinares no solo desde una validación epistemológica, sino también desde juicios axiológicos sobre la legitimidad de estos conocimientos y de las prácticas administrativas en el momento histórico actual.

- Las capacidades en comprensión lectora contribuyen a la formación de lo que Aktouf denomina una "Comprensión Crítica Permanente" en los administradores, y con ello, a una mejor interpretación de los contextos, dinámicas y agentes en los que se desenvuelven las organizaciones dado que esta capacidad desarrolla, además de competen- 
cias epistemológicas, procesos de reflexión y crítica que ejercitan la dimensión axiológica (ética y valores), los cuales contribuirán a su vez a una mejor toma de decisiones por parte de los administradores.

- Lo anterior nos lleva a concluir que la lectura y sus procesos asociados representan medios para la formación de una "comprensión crítica permanente" en los administradores que les permita realizar una gestión de las organizaciones que reconozca mejor a sus actores e interrelaciones en contextos cada vez más globales y complejos.

\section{REFERENCIAS}

(1) AKTOUF, O. (2000). Administración y Pedagogía. Colombia. Fondo Editorial Universidad EAFIT.

(2) BEDARD, R. (2003). Los Fundamentos del Pensamiento y las Prácticas Administrativas - El Rombo y las cuatro Dimensiones Filosóficas. AD-MINISTER Universidad EAFIT, Número 3 jun-dic.

(3) BERNAL, R. y MARIÑO J. (2013) Sistema Nacional de Evaluación Estandarizada de la Educación - Alineación del examen SABER 11․ Dirección de Regulación, Planeación, Estandarización y Normalización - ICFES.

(4) DANE (2008) Encuesta de Consumo Cultural 2007 - Informe de resultados. Dirección de Regulación, Planeación, Estandarización y Normalización - DIRPEN.

(5) ECHEVARRÍA, M. \& GASTÓN, I. (2000). Dificultades de comprensión lectora en estudiantes universitarios. Implicaciones en el diseño de programas de intervención, Revista Psicodidáctica, No 010.

(6) FIERRO, C. \& CARBAJAL, P. (2003). El docente y los valores desde su práctica. Revista Sinéctica, febrero-julio.

(7) LERNER, D. (1985). La relatividad de la enseñanza y la relatividad de la comprensión. Lectura y Vida, Año $6 \mathrm{~N}^{\circ} 4$, diciembre.

(8) Ministerio de CULTURA - ESPAÑA (2008). Introducción. En Gobierno de España (Ed.). Balance del Plan de Fomento de la Lectura 2004 - 2007. España.

(9) MINISTERIO DE CULTURA DE ESPAÑA (2007). Hábitos de lectura y compra de libros. En Gobierno de España (Ed.). Balance del Plan de Fomento de la Lectura 2004 - 2007. Ministerio de Cultura de España - España.

(10) MORALES, O. (2002). ¿Cómo contribuir con el desarrollo de las competencias de los estudiantes universitarios como productores de textos? Educere, enero-marzo, año/vol. 5 número 016 pp. 385-389.

(11) PAREDES, J. (2006). Decodificación y lectura. Revista electrónica Actualidades Investigativas en Educación, mayo-agosto 2006, año/vol. 6 número 002.

(12) REBOUL, O. (1992). Los valores de la educación. España. Idea Universitaria.

(13) SOLÉ, I. (1998). Estrategias de Lectura. Barcelona, España. Editorial Graó.

(14) ZEVALLOS, M. y CANO, C. (2011) Diagnóstico del Nivel de lectura en los estudiantes del programa de Administración de Empresas de la UJTL. Resultado de investigación no publicado. Universidad de Bogotá Jorge Tadeo Lozano, Bogotá, Colombia. 